\title{
DNA binding by Corynebacterium glutamicum TetR-type transcription regulator AmtR Daniela Muhl ${ }^{1}$, Nadja Jeßberger ${ }^{1}$, Kristin Hasselt ${ }^{1}$, Christophe Jardin ${ }^{2}$, Heinrich Sticht ${ }^{2}$ and Andreas Burkovski*1
}

\begin{abstract}
Address: ${ }^{1}$ Lehrstuhl für Mikrobiologie, Friedrich-Alexander-Universität Erlangen-Nürnberg, Erlangen, Germany and ${ }^{2}$ Institut für Biochemie, Friedrich-Alexander-Universität Erlangen-Nürnberg, Erlangen, Germany

Email: Daniela Muhl -dmuhl@biologie.uni-erlangen.de; Nadja Jeßberger - njessber@biologie.uni-erlangen.de; Kristin Hasselt - khasselt@biologie.uni-erlangen.de; Christophe Jardin - christophe.jardin@biochem.uni-erlangen.de; Heinrich Sticht - Heinrich.Sticht@med.uni-erlangen.de; Andreas Burkovski* - aburkov@biologie.uni-erlangen.de

* Corresponding author
\end{abstract}

Published: 23 July 2009

BMC Molecular Biology 2009, 10:73 doi:10.1 186/1471-2199-10-73

This article is available from: http://www.biomedcentral.com/147/-2/99/10/73

(C) 2009 Muhl et al; licensee BioMed Central Ltd.

This is an Open Access article distributed under the terms of the Creative Commons Attribution License (http://creativecommons.org/licenses/by/2.0), which permits unrestricted use, distribution, and reproduction in any medium, provided the original work is properly cited.
Received: 23 April 2009

Accepted: 23 July 2009

\begin{abstract}
Background: The TetR family member AmtR is the central regulator of nitrogen starvation response in Corynebacterium glutamicum. While the AmtR regulon was physiologically characterized in great detail up to now, mechanistic questions of AmtR binding were not addressed. This study presents a characterization of functionally important amino acids in the DNA binding domain of AmtR and of crucial nucleotides in the AmtR recognition motif.

Results: Site-directed mutagenesis, the characterization of corresponding mutant proteins by gel retardation assays and surface plasmon resonance and molecular modelling revealed several amino acids, which are directly involved in DNA binding, while others have more structural function. Furthermore, we could show that the spacing of the binding motif half sites is crucial for repression of transcription by AmtR.

Conclusion: Although the DNA binding domain of TetR-type repressors is highly conserved and a core binding motif was identified for AmtR and TetR(D), the AmtR binding domain shows individual properties compared to other TetR proteins. Besides by distinct amino acids of AmtR, DNA binding is influenced by nucleotides not only of the conserved binding motif but also by spacing nucleotides in C. glutamicum.
\end{abstract}

\section{Background}

Almost all of the macromolecules in a bacterial cell, e.g. proteins, nucleic acids and cell wall components, contain nitrogen. Thus, prokaryotes have developed elaborate mechanisms to provide an optimal nitrogen supply for metabolism and to overcome and survive situations of nitrogen limitation, generally summarized as nitrogen control. This communication focuses on nitrogen control in Corynebacterium glutamicum, a Gram-positive soil bacte- rium used for the industrial production of amino acids [1]. We have been studying nitrogen metabolism and nitrogen regulation in corynebacteria with a focus on $C$. glutamicum for several years (for recent reviews, see [2-4]) and could show that transcription of genes in response to nitrogen limitation is governed in corynebacteria, i.e. $C$. glutamicum, Corynebacterium efficiens and Corynebacterium diphtheriae, by TetR-type regulator AmtR [5,6], which blocks transcription of various genes during growth in 
nitrogen-rich medium. The AmtR regulon of C. glutamicum was characterized by a combination of bioinformatics and molecular biology approaches. At least 35 genes, which encode transporters and enzymes for ammonium assimilation $(a m t A, a m t B, g \ln A, g l t B D, d a p D)$, creatinine $(\operatorname{cod} A, c r n T)$ and urea metabolism (urtABCDE, ureABCE$F G D$ ), a number of biochemically uncharacterized enzymes and transport systems as well as signal transduction proteins $(g \ln D, g \ln K)$, are directly controlled by the AmtR protein in C. glutamicum $[7,8]$.

An AmtR binding site consensus motif was deduced from bioinformatic analyses of available genome sequence information and competitive gel retardation assays $[7,8]$. The resulting AmtR box with the nucleotide sequence ttCTATN ${ }_{6}$ AtAGat/aA (with bases represented by capital letters being highly conserved) is a more or less palindromic sequence and can be located in the promoter region either on the sense or antisense strand.

In this study we address the question which amino acids within the AmtR DNA binding domain are in fact contacting the DNA and why AmtR expression is not controlled by an autoregulatory circuit as found for other TetR-type regulators (for review, see [9])

\section{Results}

Characterization of the AmtR binding domain reveals functionally important amino acid residues

As a molecular biology approach to identify amino acid residues of AmtR involved in DNA binding, site-directed mutagenesis experiments were carried out. Amino acids highly conserved in the DNA binding domain of TetR family proteins (Fig. 1) were selected and exchanged against alanine, with exception of the conserved Ala54 residue, which was changed to glycine. Wild type AmtR and AmtR variants were purified as maltose binding protein (MBP) fusions and applied in gel retardation experiments. As target sequence a PCR fragment spanning nucleotides -298 to -1 relative to the start codon of the $a m t B$ gene and comprising three AmtR binding sites [4,7] was used. While $150 \mathrm{ng}$ of wild type AmtR-MBP led to a complete retardation of $0.04 \mathrm{ng}$ of target DNA, the same amount of MBP had no effect (Fig. 2A). Subsequently carried out gel retardation experiments with rising amounts of AmtR variants (addition of AmtR*-MBP up to $3 \mu \mathrm{g}$ ) revealed that exchange of residues Glu23, Thr33, Gly36 and Thr42 had no effect. The corresponding recombinant proteins behaved as wild type. A slightly reduced affinity compared to wild type AmtR was observed for AmtR* with exchange of His43 and Arg52 to alanine and Ala54 to glycine, while significantly reduced binding was observed for alterations of Glu30, Leu31, Thr40, Gly50, Gln53, Ser55, Tyr57, Tyr58 and Leu71. Mutations resulting in an alanine exchange at positions Phe32, Ile51, Leu56, His59 and Leu70 led to a complete loss of AmtR* binding (Fig. 2B).

From these gel retardation assays, $\mathrm{K}_{\mathrm{D}}$ values for the binding of AmtR-MBP and selected AmtR*-MBP variants were estimated. The equilibrium binding constant for AmtR$\mathrm{MBP}$ was $2.4 \times 10^{-6} \mathrm{M}$ and $2.2 \times 10^{-6} \mathrm{M}$ for the variant car-

\footnotetext{
C. glutamicum

C. efficiens

C. diphtheriae

s. avermitilis

M. smegmatis

N. farcinica

A. aurescens

Rhodococcus sp.

C. glutamicum

C. efficiens

C. diphtheriae

s. avermitilis

M. smegmatis

N. farcinica

A. aurescens

Rhodococcus sp.

C. glutamicum

C. efficiens

C. diphtheriae

$S$. avermitilis

M. smegmatis

N. farcinica

A. aurescens

Rhodococcus sp.
}

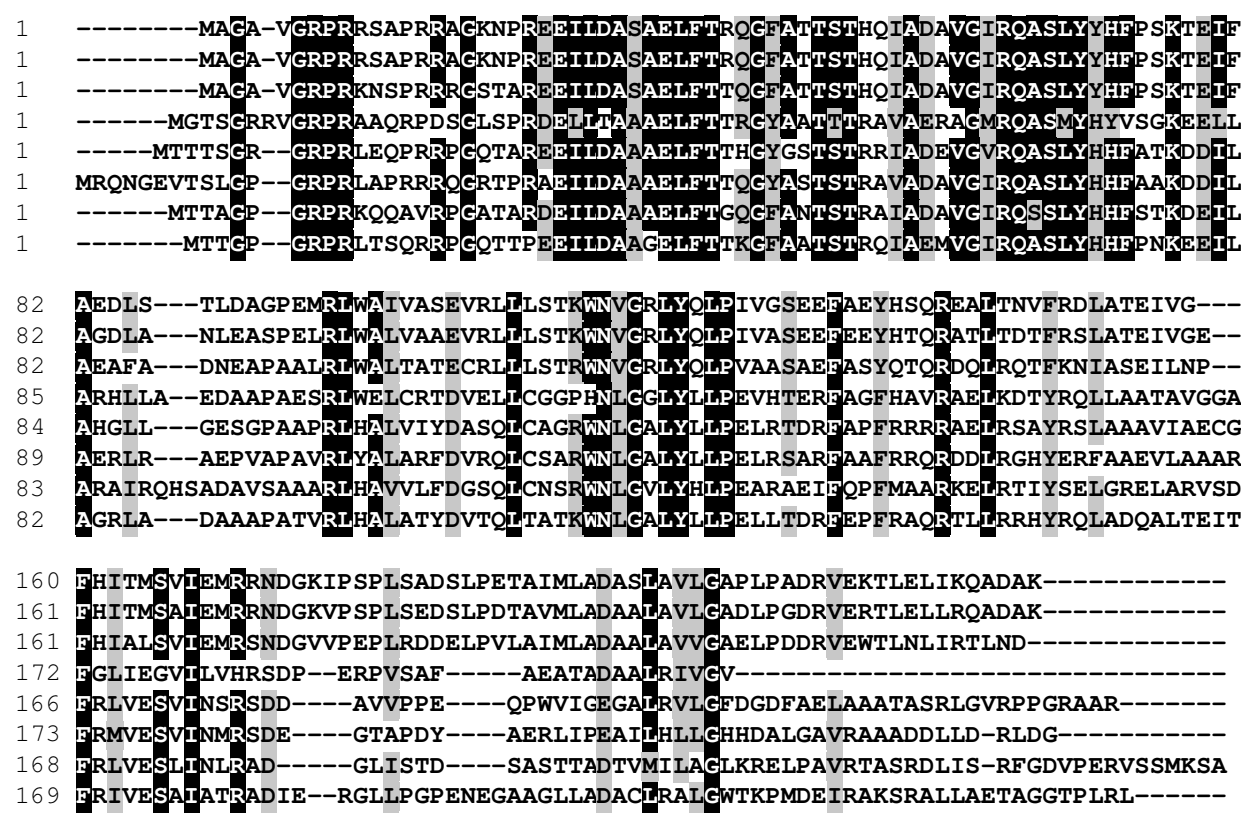

Figure I

Sequence alignment of AmtR proteins from different Gram-positive bacteria. Amino acid residues identical in all sequences are shaded in black, other conserved amino acids in gray. 

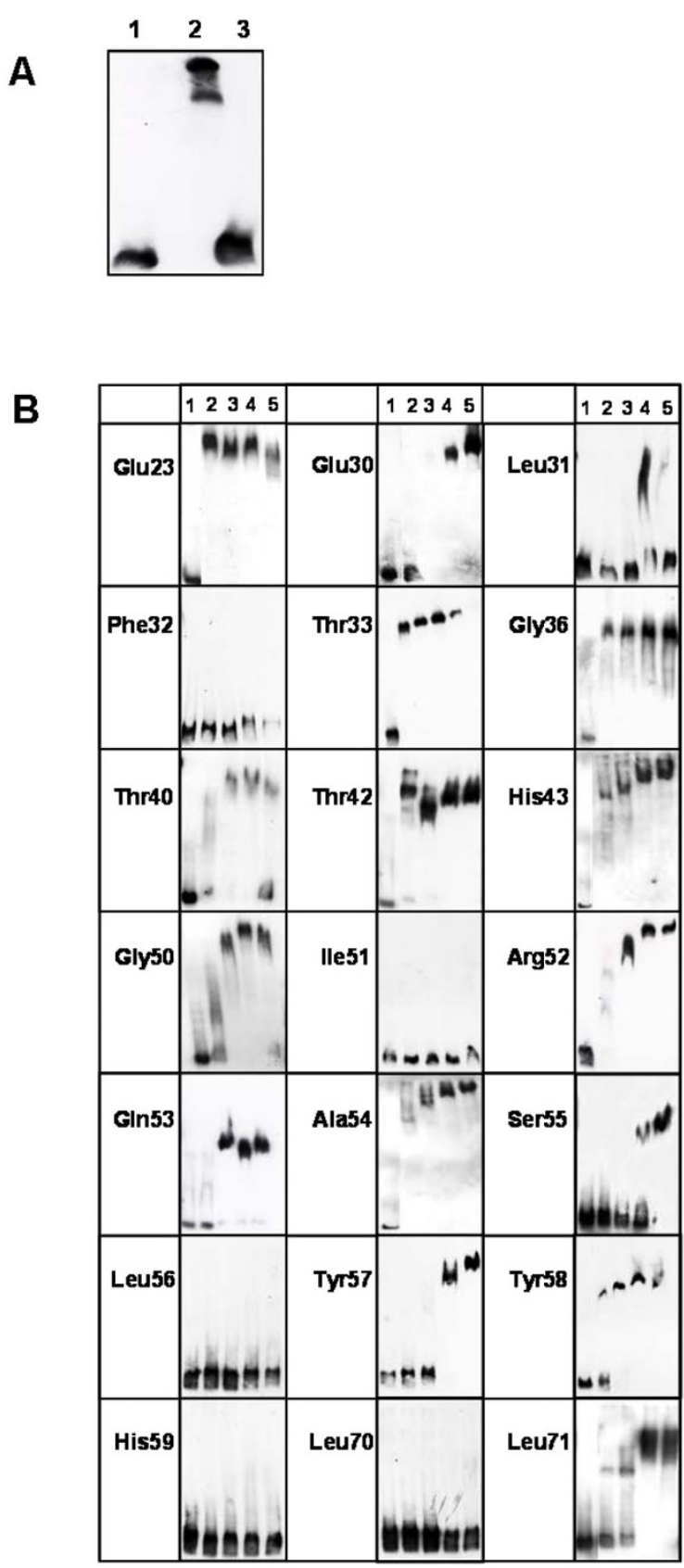

Figure 2

Gelretardation assays using AmtR variants. (A) $A$ DNA fragment spanning nucleotides -298 to - I relative to the start codon of $a m t B(0.04 \mathrm{ng}$ DNA per lane) was used for the gel shift assay (I) negative control: $50 \mathrm{ng}(2170 \mathrm{nM})$ of MBP, (2) $150 \mathrm{ng}$ (2I70 nM) wild type AmtR fused to MBP, (3) $a m t B$ upstream DNA without added protein. (B) Recombinant AmtR-MBP proteins carrying alanine exchanges of the indicated amino acid residues (with exception of Ala54, which was altered to glycine). DNA as described above plus (I) $0 \mathrm{ng}$, (2) $150 \mathrm{ng}$, (3) $750 \mathrm{ng}$, (4) $1950 \mathrm{ng}$, (5) $3 \mu \mathrm{g}$ of the indicated AmtR*-MBP fusion. rying the Thr33Ala exchange, which is in accordance with the wild type-like behaviour of this protein in the gel retardation assay. With rising effect on binding, increasing $\mathrm{K}_{\mathrm{D}}$ values were estimated, e. g. 1.0 and $2.0 \times 10^{-5} \mathrm{M}$ for exchange Glu30 and Leu71, as well as $7.1 \times 10^{-5} \mathrm{M}$ for AmtR*-MBP carrying the Ser55Ala exchange.

As an independent assay, surface plasmon resonance (SPR) measurements were carried out. In these experiments, the same AmtR-MBP preparations as in the gel retardation assays were used, while the DNA immobilized on the chip surface corresponded to a shorter fragment of the $a m t B$ promoter resembling only one AmtR binding site (nucleotide position -186 to -156, [4]). Again, purified maltose binding protein was used as negative control and AmtR-MBP as positive control (Fig. 3A). MBP did not bind to the immobilized DNA, while addition of AmtRMBP resulted in a clearly dose-dependent increase in response units, indicating binding of the protein to its target DNA. Similarly, binding of AmtR variants was tested (Fig. 3B). In general, results obtained with this approach were similar to that described above for the gel retardation assays. However, for exchange of Thr42, Ser55, Tyr57 and Tyr58 a stronger reduction of binding was observed, which might indicate a stabilization effect or cooperative binding of AmtR-MBP, when more than one binding site is available.

In summary, the analysis of AmtR variants generated by site-directed mutagenesis and analyzed by gel retardation experiments and SPR measurements hint to a crucial role of several amino acids in DNA binding. However, it was difficult to differ between direct and indirect effects. Therefore, modelling experiments were carried out.

\section{Molecular modelling indicates the function of distinct amino acids}

In order to allow a structural interpretation of the AmtR mutation data, we started a molecular modeling approach of AmtR in complex with DNA based on the crystal structure of the TetR-DNA complex. The significant sequence identity between the DNA-binding domains of the proteins and the fact that they recognize an identical "CTAT" core motif allowed the calculation of a molecular model (Fig. 4), which provides the basis for subsequent detailed analysis.

The model shares the same characteristic structural properties previously reported for the TetR-DNA crystal structure: The DNA-binding domains are constituted of helices $\alpha 1$ to $\alpha 4$ of the $\mathrm{N}$-terminal domains. The binding motif itself consists of the $\alpha 2-\alpha 3$ loops (amino acid residues Thr42 to His59) organized in helix-turn-helix (HTH) motifs whereas helix 4 constitutes the link between the $\mathrm{N}$ - 
A

MBP

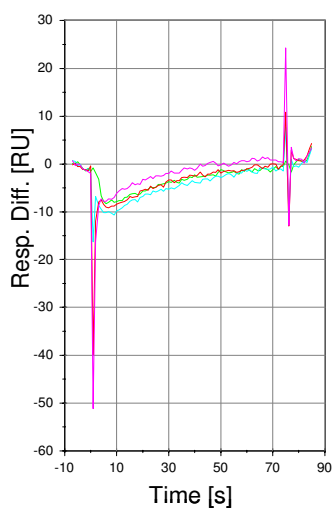

AmtR-MBP

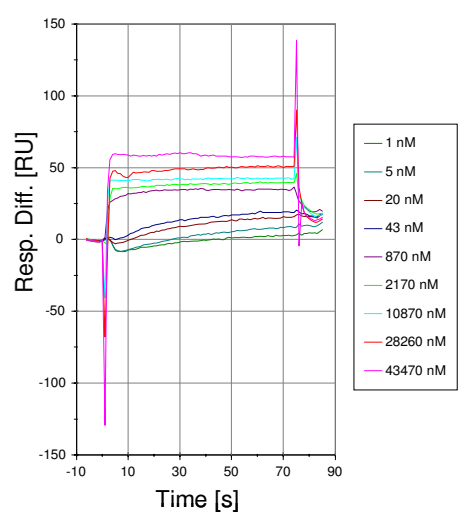

\section{B}

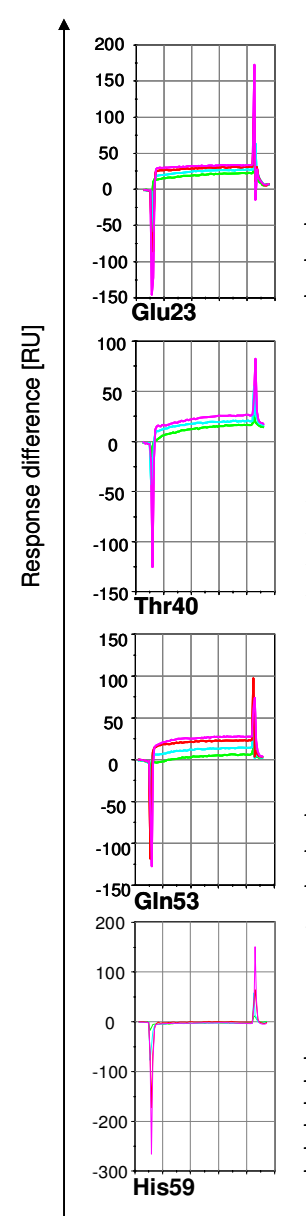

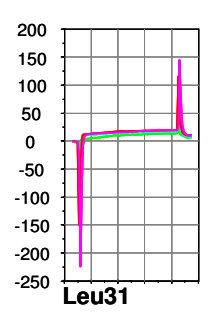
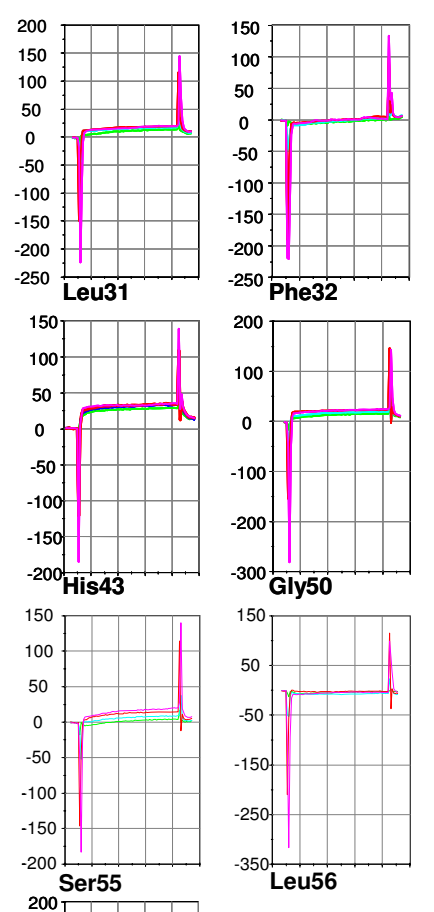

-300 Gly50

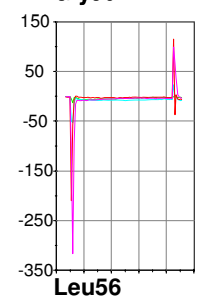

Ala54
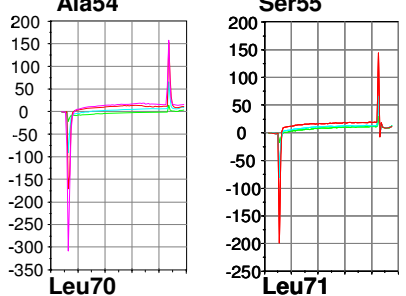
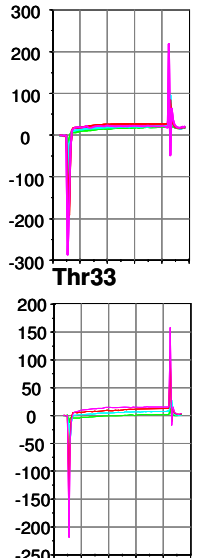

Ile51

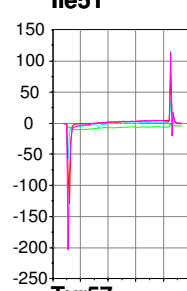

Tyr57

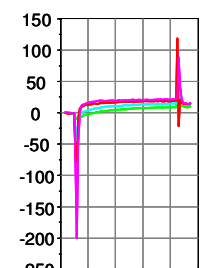

Gly36

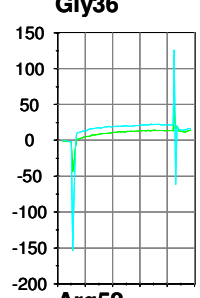

Arg52

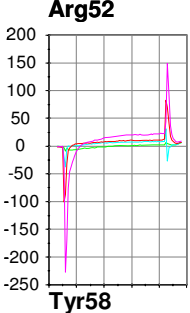

Time [s]

Figure 3

Surface plasmon resonance measurements. An amtB promoter fragment spanning nucleotides - I86 to - I56 (relative to the start codon) was immobilized on Biacore chips rising concentrations of protein were added (for colour code, see Fig. $3 \mathrm{~A}$ ). (A) Binding properties of negative control (MBP) and positive control (AmtR-MBP fusion), (B) Influence of amino acid exchanges on binding of AmtR variants. Colour code for the concentrations of proteins added as in (A). 

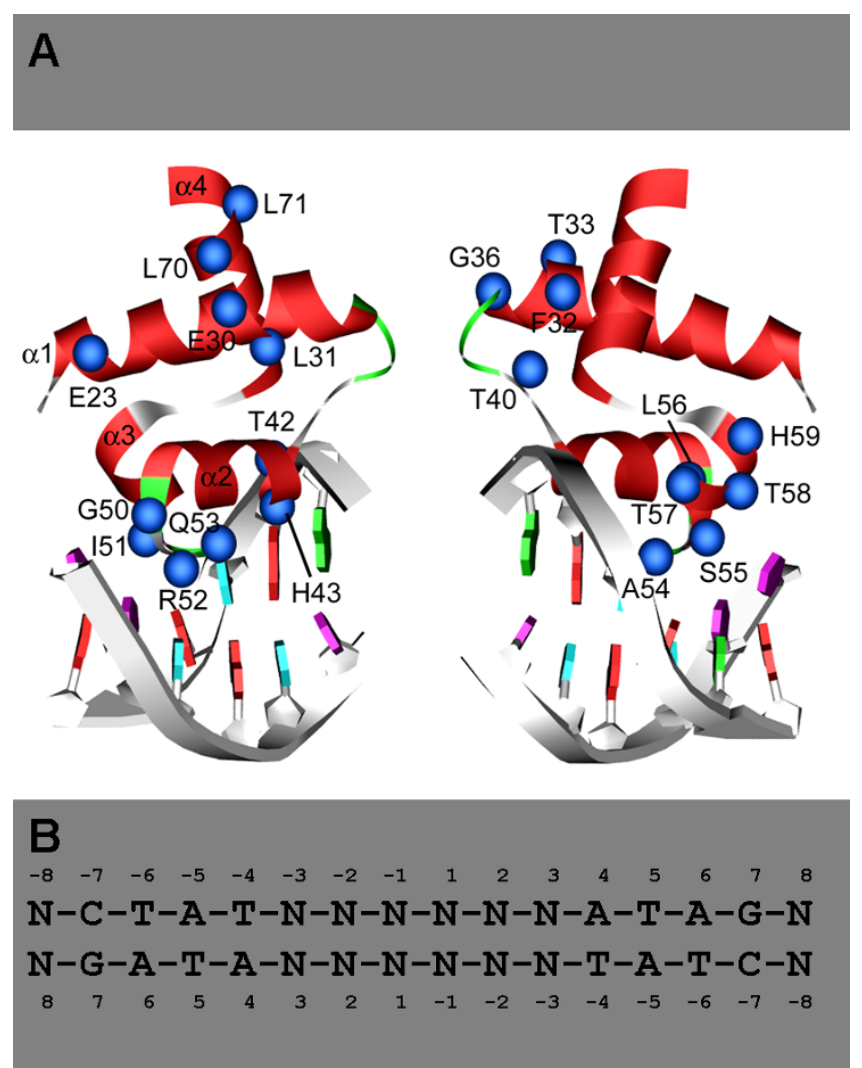

Figure 4

Homology model of the AmtR repressor-operator complex. (A) Three-dimensional model of AmtR DNA binding domain in complex with DNA. The two binding sites of dimeric AmtR are shown separately on the left and right

half of the picture. The protein is depicted in backbone presentation and sequence positions that were experimentally investigated are shown as balls. The DNA backbone is shown as grey ribbon and the bases are colored according to their type. (B) Sequence of the AmtR operator indicating the numbering scheme used in the present work. The bases of the conserved "CTAT" recognition motif are explicitly labeled, while the remaining nonconserved bases are denoted as "N".

and C-terminal domains. Each HTH motif of the repressor binds to one major groove of the palindromic DNA consensus nucleotide sequence "CTAT". Due to this twofold symmetry, subsequent bioinformatic analysis was restricted to one half-site of the complex.

The AmtR-DNA model complex reveals that 9 of the 21 residues investigated are in contact with the DNA while the remaining 12 residues are located within the HTH binding motif but do not directly bind DNA (Fig. 4; Table 1 ). These two groups were termed interface (IF) and noninterface (NI) residues and their functions are discussed separately below. For several non-interface residues (Glu23, Thr33, Gly36), replacement by alanine had no effect on DNA binding properties. Structural analysis reveals that these residues are solvent exposed and do not form crucial interactions within AmtR (Fig. 5a).

A completely different situation is present for those noninterface residues, for which replacement by alanine leads to a complete loss of DNA binding (Phe32, Ile51, Leu56, Leu70). These residues are buried within the interior of the protein and form interactions that stabilize the tertiary structure of the HTH motif (Table 1; Fig. 5A, B). The respective interactions cannot be formed by alanine in the mutant protein structures, which will lead to a disruption of the three-dimensional structure of the binding domains consequently resulting in a (complete) loss of DNA binding activity.

For the remaining group of non-interface residues (Glu30, Leu31, Thr40, Gly50, Leu71), mutation results in weaker DNA binding. These residues are either buried in the protein structure (Leu31, Thr40, Leu71), or involved in the formation of a tight turn (Gly50) or a salt-bridge (Glu30). A replacement by alanine is therefore expected to have at least a moderate destabilizing effect for all of these sequence positions. The mechanism, by which this destabilization will reduce DNA binding affinity, cannot unambiguously be determined from the static model structure. One might speculate that mutation either leads to rearrangements within AmtR or that mutation might increase the portion of unfolded HTH domains, which are no longer capable of DNA binding. The observation that mutations can affect the disorder-order equilibrium of proteins has been recently also described for a mutation within the DNA binding domain of TetR as well [10].

The nine interface residues investigated can be divided into those, which have no or moderate effects on binding affinity (Thr42, His43, Arg52, Ala54) and those which have a strong effect (Gln53, Ser55, Tyr57, Tyr58, His59). The first group of residues generally forms only weak interactions with the DNA (Table 1; Fig. 5C, D), while Gln53, Ser55, and Tyr57 form tight contacts with the "CTAT"-motif (Table 1; Fig. 5E). Gln53 for example specifically recognizes the purine ring of $A 4$ via two hydrogen bonds.

A special situation is observed for residues Tyr58 and His59, which form only weak contacts with the DNA, but nevertheless have a strong effect on binding affinity when mutated to alanine: Tyr58 forms only weak interactions with the ring of a non-conserved nucleotide at promoter position -8 that is located adjacent to the "CTAT"-motif (Fig. 5F). Tyr58, however, might play an additional role for stabilizing the HTH motif by interaction with His59. The side chain orientation of His59 appears to be particularly important, since this residue forms both a hydrogen bond with Asn19 at the N-terminus of helix $\alpha 1$, as well as a water-mediated interaction with a phosphoryl group 

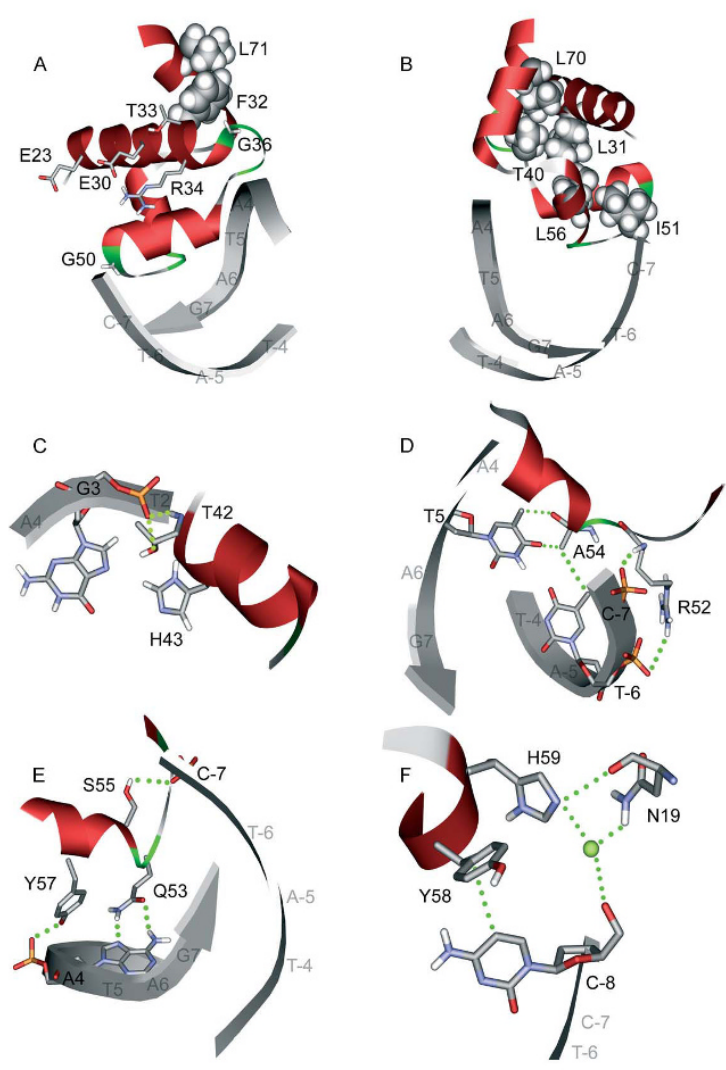

\section{Figure 5}

Detailed structural analysis of different residues in the DNA binding domain of AmtR. Panels (A) and (B): Location and interactions of the non-interface residues that were experimentally investigated. Surface-exposed residues are depicted as sticks, while those residues that are buried in the interior of the protein are shown in space-filled presentation. Panels (C) to (F): Interactions of the residues located in the protein DNA interface. The DNA backbone is shown as grey tube and nucleotides of the binding site are shown in stick presentation. Contacts are indicated by dotted lines.

The residues shown in (F) might play a dual role by forming both contacts with the DNA and with other parts of the protein. A water molecule, which was modeled in analogy to the TetR-operator complex crystal structure, is shown as green ball. See text for more details.

(Fig. 5F) that was deduced in analogy to the TetR crystal structure. Mutation of His59 to alanine might therefore affect DNA binding both by direct and by indirect effects thereby explaining the strong influence of this mutation.

The lack of AmtR autoregulation is caused by variations of spacing nucleotides rather than variation of the core binding motif

Compared to other TetR family members, C. glutamicum AmtR lacks an autoregulation circuit (for review, see [9]). Neither with DNA microarrays nor with real-time reverse transcriptase PCR an upregulation of amtR expression upon nitrogen starvation was detectable in different studies [7,8,11-13], although an AmtR consensus site was identified upstream of the amtR gene [5]. The reason for the lack of autoregulation was unclear until now, however, compared to other AmtR binding sites the amtR upstream sequence differs in two points (i) the presence of a $\mathrm{G}$ instead of $\mathrm{T}$ at position 2 of the core motif and (ii) in the number of spacing nucleotides between the two half sites, 3 instead of 4 . To investigate the importance of these differences, gel shift experiments were carried out using AmtR-MBP, wild type and modified $a m t R$ promoter regions (Fig. 6). While a $\mathrm{G}$ to $\mathrm{T}$ nucleotide exchange at position 2 had no significant effect, the introduction of an additional nucleotide clearly led to the binding of AmtRMBP.

\section{Discussion}

TetR-type regulators are widely distributed among Grampositive bacteria [9] including corynebacteria. In fact, genome sequence analyses of Corynebacterium diphtheriae, Corynebacterium efficiens, C. glutamicum and Corynebacterium jeikeium revealed that these are the most frequently used transcriptional regulators in corynebacteria [13]. Examples for functionally characterized members of the TetR family in C. glutamicum besides AmtR are AcnR, CGL2612 and McbR. AcnR binds upstream the acn gene encoding aconitase to the putative consensus sequence CAGNACnnncGTACTG, which is highly conserved in corynebacterial and mycobacterial species [14]. CGL2612 is a drug resistance-related regulator with significant structural similarity to the multidrug resistance-related transcription factor QacR from Staphylococcus aureus [15]. McbR binds to the consensus motif TAGAC-N6-GTCTA and is involved in the regulation of sulphur metabolism and the synthesis of sulphur-containing amino acids $[16,17]$.

Compared to the other TetR-family members in $C$. glutamicum, AcnR [14] and CGL2612 [15], the AmtR regulon is relatively big and compared to especially McbR [17], the binding consensus is not very strictly conserved. This raises the questions about the similarities and differences in protein-DNA recognition, especially in respect to the well-investigated E. coli TetR. For the AmtR proteinDNA interface, mutagenesis revealed an important role for Thr42, Gln53, Ser55, Tyr57, Tyr58, and His59. These residues are either strictly conserved or there are only very conservative replacements observed between AmtR and TetR (Table 1). This finding is consistent with the fact that both repressors recognize the identical "CTAT"-motif in the DNA.

For the remaining three residues of the protein-DNA interface (His43, Arg52, Ala54) differences are observed between AmtR and TetR, which might have implications 
Table I: Structural features and role for DNA binding of different AmtR residues.

\begin{tabular}{|c|c|c|c|c|}
\hline Sequence position & Effect on DNA binding & Location & Structural features & Equivalent residue in E. coli TetR \\
\hline Glu23 & o & $\mathrm{NI}$ & solvent exposed, only few interactions & Ser8 \\
\hline Glu30 & ++ & $\mathrm{NI}$ & Glu30-Arg34 salt bridge & Glul5 \\
\hline Leu3। & ++ & $\mathrm{NI}$ & buried & Leul6 \\
\hline Phe32 & +++ & $\mathrm{NI}$ & buried & Leul7 \\
\hline Thr33 & ○ & $\mathrm{NI}$ & solvent exposed, only few interactions & Asn 18 \\
\hline Gly36 & $\circ$ & $\mathrm{NI}$ & $\begin{array}{l}\text { C-terminus of helix } \alpha \text { l; only few } \\
\text { interactions }\end{array}$ & Gly2I \\
\hline Thr40 & ++ & $\mathrm{NI}$ & buried & Leu25 \\
\hline Thr42 & $\mathrm{o}(+)$ & IF & $\begin{array}{l}\text { backbone interactions with } G 3 \text { phosphate } \\
\text { group }\end{array}$ & Thr27 \\
\hline His43 & + & IF & G3 ring & $\operatorname{Arg} 28$ \\
\hline Gly50 & ++ & $\mathrm{NI}$ & tight turn & Gly35 \\
\hline |le5। & +++ & $\mathrm{NI}$ & buried & lle36 \\
\hline $\operatorname{Arg} 52$ & + & IF & $\begin{array}{l}\text { backbone contact with } T-6 \text { phosphate } \\
\text { group }\end{array}$ & Glu37 \\
\hline $\mathrm{G} \ln 53$ & ++ & IF & side chain contacts with $A 4$ ring & $\mathrm{G} \ln 38$ \\
\hline Ala54 & + & $\mathrm{IF}$ & $\begin{array}{l}\text { weak non-polar interactions with the } \\
\text { methyl group of } T-6\end{array}$ & Pro39 \\
\hline Ser55 & $++(+)$ & IF & side chain contacts with $C-7$ & Thr40 \\
\hline Leu56 & +++ & $\mathrm{NI}$ & buried & Leu4I \\
\hline Tyr57 & $++(+)$ & IF & side chain contacts with $A 4$ and $T 5$ & Tyr42 \\
\hline Tyr58 & $++(+)$ & IF & $\begin{array}{l}\text { side chain interactions with DNA and with } \\
\text { His59 }\end{array}$ & Trp43 \\
\hline His59 & +++ & IF & $\begin{array}{l}\text { side chain hydrogen bonds to protein and } \\
\text { DNA }\end{array}$ & His44 \\
\hline Leu70 & +++ & $\mathrm{NI}$ & buried & Leu55 \\
\hline Leu7। & ++ & $\mathrm{NI}$ & partially buried & Ala56 \\
\hline
\end{tabular}

The first and second column list the residues investigated and their experimentally determined effect on DNA binding. "o" indicates no effect, while increasing numbers of "+ signs qualitatively reflect the magnitude, by which DNA binding is decreased. Signs in parenthesis indicate minor differences between the two experimental methods used. The third and fourth column list the location of the residue in the structure as deduced from the AmtR-DNA model as well as key structural features that might be of relevance for DNA binding. "IF" and "NI" denote protein-DNA interface and non-interface residues, respectively. The last column shows the structurally equivalent residues in $E$. coli TetR. 


\section{$\begin{array}{lllllllll}1 & 2 & 3 & 4 & 5 & 6 & 7 & 8 & 9\end{array}$}

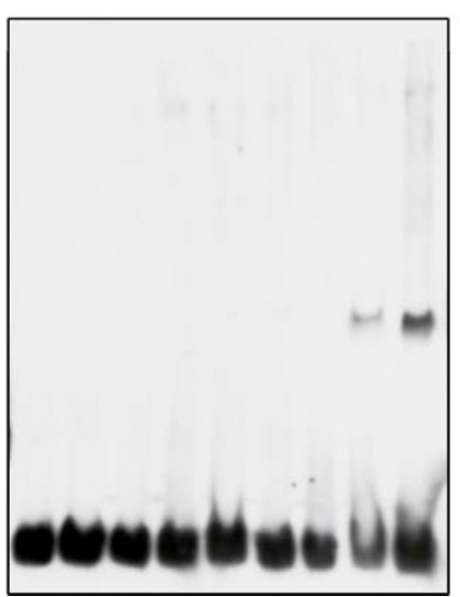

Figure 6

Gelretardation assays using amtR upstream DNA variants. (I-3) $0.04 \mathrm{ng}$ of native DNA, (4-6) amtR upstream DNA carrying a $G$ to $T$ exchange at position 2, (7-9) amtR upstream DNA with additional spacing nucleotide. $(1,4,7)$ without AmtR-MBP, $(2,5,8)$ with I50, $(3,6,9)$ with $300 \mathrm{ng}$ of AmtR-MBP.

for binding affinity and specificity. His43 is replaced by arginine in TetR. This arginine forms specific contacts with the purine ring of G3 in the TetR-DNA crystal structure [18]. In the model of the AmtR-DNA complex, the distance between His43 and the purine ring is larger due to the shorter histidine side chain, suggesting a weaker interaction (Fig. 5C). Although these differences might also arise from inaccuracies of our model, there are two observations which suggest a real difference between AmtR and TetR. First, a replacement of His 43 by alanine leads only to a minor decrease of binding affinity (Fig. 2,3). Second, the respective G-C base pair does not represent a part of the "CTAT" core motif in AmtR (Fig. 4B) and is non-conserved in AmtR binding sequences [7], while it is conserved in the "CTATC"-motif recognized by TetR. Arg52 of AmtR is replaced by glutamic acid in TetR. The presence of the non-conservative replacement together with the observation that an arginine to alanine replacement has only little effect on AmtR binding affinity suggest that the Arg52 side chain does not play a major role for the DNA interaction.

Another difference is observed at position 54: The respective alanine of AmtR is equivalent to Pro39 of TetR. Ala 54 forms interactions with the methyl group of $T-6$. These interactions, however, seem only to play a minor role for the affinity and specificity of DNA recognition. This is evidenced by the observation that a replacement by glycine has only small effects on binding affinity. In addition, the contact to the methyl-group of base T- 6 seems not to mediate a specific recognition since a replacement of thymidine by guanosine still allows an interaction (Fig. 5D, Fig. 6). In contrast, Pro39 of TetR contacts two thymidines of adjacent base pairs (T5 and T-6 according to the $a m t R$ nomenclature; Fig. 4B) and a replacement by glutamate was shown to have a significant effect on binding affinity and specificity [19]. These findings suggest that Ala54 of AmtR has a smaller role than Pro39 in TetR for promoter recognition.

In summary, at least the differences at sequence positions 43 and 54 might explain the larger sequence variability of DNA recognition by AmtR compared to TetR. Although AmtR tolerates a certain amount of variability within the DNA half-sites, their correct spacing appears to be crucial for binding. The "CTAT" half-sites are separated by a fiveresidue spacer in TetR, but by six residues in AmtR. As demonstrated by the experiment in Fig. 6, this spacing is essential for the AmtR-DNA interaction. Due to the differences of the spacer length between TetR and AmtR, the structural consequences of this feature on the orientation of the binding heads or the curvature of the DNA cannot be deduced from the present model, but have to await the elucidation of a crystal structure of AmtR in complex with DNA. Future work will now concentrate on the crystallization of AmtR without and together with bound DNA. Using DNA fragments with alterations of the consensus motif and the kind and number of spacing nucleotides, this approach will allow to study functional and structural flexibility of DNA binding domains. Furthermore, the cocrystallization of AmtR with signal transduction protein GlnK will provide the first structural information about an asymmetric GlnK interaction complex. Today, besides the interaction with AmtR, which most likely acts as a dimer [5], exclusively interactions with threefold symmetry were reported, e.g. of the trimeric GlnK complex with trimeric ammonium transporter $\mathrm{AmtB}$, or with the hexameric key enzyme for arginine biosynthesis NAGK [20].

\section{Conclusion}

Molecular biology and biochemistry approaches such as site-directed mutagenesis, gel retardation assays and SPR are, especially in combination with molecular modelling, powerful tools to identify crucial amino acids for DNA binding. In this study, we could show that besides by distinct amino acids of the TetR family protein AmtR, DNA binding is influenced by nucleotides not only of the conserved binding motif but also by spacing nucleotides in $C$. glutamicum.

\section{Methods}

Bacterial strains and growth

C. glutamicum wild type ATCC 13032 [21] was grown at $30^{\circ} \mathrm{C}$ in MOPS-buffered minimal medium with glucose as carbon source as described [22]. 
Table 2: Oligonucleotides used in this study.

\begin{tabular}{|c|c|c|}
\hline Designation & Sequence $\left(5^{\prime} \rightarrow 3^{\prime}\right)$ & Application \\
\hline amtRw/oATG-BamHI-fw & GGTCGGATCCGCAGGAGCAGTGGG & Cloning of $a m t R$ into pMalc2 \\
\hline amtR-Pstl-rev & GGCGCCTGCAGTTATTTCGCGTCAGCCTGC & Cloning of $a m t R$ into pMalc2 \\
\hline amtR23-fwd & TCCTCGCGAGGCGATTCTTGACG & Mutagenesis of $a m t R$ \\
\hline amtR25-fwd & CGAGGAGATTGCTGACGCCTCTG & Mutagenesis of $a m t R$ \\
\hline amtR30-fwd & CGCCTCTGCTGCGCTTTTCACCC & Mutagenesis of $a m t R$ \\
\hline amtR3I-fwd & СTCTGCTGAGGCTTTCACCCGTC & Mutagenesis of $a m t R$ \\
\hline amtR32-fwd & TGCTGAGCTTGCCACCCGTCAAG & Mutagenesis of $a m t R$ \\
\hline amtR33-fwd & TGAGCTTTTCGCCCGTCAAGGCT & Mutagenesis of $a m t R$ \\
\hline amtR36-fwd & CACCCGTCAAGCCTTCGCAACAA & Mutagenesis of $a m t R$ \\
\hline amtR40-fwd & CTTCGCAACAGCCTCCACGCATC & Mutagenesis of $a m t R$ \\
\hline amtR42-fwd & AACAACCTCCGCGCATCAAATCG & Mutagenesis of $a m t R$ \\
\hline amtRH43A & CAACCTCCACGGCTCAAATCGCTG & Mutagenesis of $a m t R$ \\
\hline amtR50-fwd & TGATGCCGTGGCAATCCGCCAAG & Mutagenesis of $a m t R$ \\
\hline amtR5I-fwd & TGCCGTGGGAGCCCGCCAAGCCT & Mutagenesis of $a m t R$ \\
\hline amtR52-fwd & CGTGGGAATCGCCCAAGCCTCGC & Mutagenesis of $a m t R$ \\
\hline amtR53-fwd & GGGAATCCGCGCAGCCTCGCTCT & Mutagenesis of $a m t R$ \\
\hline amtRA54A & GAATCCGCCAAGGCTCGCTGTATTATC & Mutagenesis of $a m t R$ \\
\hline amtR55-fwd & CCGCCAAGCCGCGCTGTATTATC & Mutagenesis of $a m t R$ \\
\hline amtR56-fwd & CCAAGCCTCGGCGTATTATCACT & Mutagenesis of $a m t R$ \\
\hline amtR57-fwd & AGCCTCGCTGGCTTATCACTTCC & Mutagenesis of $a m t R$ \\
\hline amtR58-fwd & CTCGCTGTATGCTCACTTCCCGT & Mutagenesis of $a m t R$ \\
\hline amtR59-fwd & GCTGTATTATGCCTTCCCGTCCA & Mutagenesis of $a m t R$ \\
\hline amtR63-fwd & CTTCCCGTCCGCGACGGAAATCT & Mutagenesis of $a m t R$ \\
\hline amtR70-fwd & CTTCCTCACCGCGCTGAAATCTA & Mutagenesis of $a m t R$ \\
\hline probe-amtB-fw & GCT GGG CTA GAA ACC CGA & $\begin{array}{l}\text { amtB promoter fragment for gel retardation assays } \\
\text { (nt position }-298 \text { to }-1 \text { ) }\end{array}$ \\
\hline probe-amtB-rev & GCG TGG ATG ACC TCC TTT G & $\begin{array}{l}\text { amtB promoter fragment for gel retardation assays } \\
\text { (nt position }-298 \text { to }-I \text { ) }\end{array}$ \\
\hline bindingI_amtB-fw & TAAATTACCTGTTAAACTATAGAAAATATC & $a m t B$ promoter fragment for SPR (nt position -186 to -156 ) \\
\hline
\end{tabular}


Table 2: Oligonucleotides used in this study. (Continued)

\begin{tabular}{lll}
\hline bindI_amtB-rew-2 & GATATTTTCTATAGTTTAACAGGTAATTTA & amtB promoter fragment for SPR (nt position - I86 to - I56) \\
\hline amtRbs-f & GCCCGTGGTGTGCTCACCAATG & $\begin{array}{l}\text { amtR promoter fragment for gel retardation assays } \\
\text { (nt position -29l to -63) }\end{array}$ \\
\hline amtRbs-r & CAGAGTTCCTATTTGGTATCGATTTCACGGGC & $\begin{array}{l}\text { amtR promoter fragment for gel retardation assays } \\
\text { (nt position -29l to -63) }\end{array}$ \\
\hline amtRbsG-T-r & CAGAGTTCCTATTTGGTATAGATTTCACGGGC & amtR promoter fragment for gel retardation assays \\
\hline amtRbs+N-r & CAGAGTTCCTATTATGGTATCGATTTCACGGGC & amtR promoter fragment for gel retardation assays \\
\hline
\end{tabular}

\section{General molecular biology techniques}

For plasmid isolation, transformation, and cloning standard techniques were used [23]. E. coli strain DH5 $\alpha \mathrm{mcr}$ [24] was used as cloning host.

\section{Site directed mutagenesis}

For expression and purification of AmtR variants, point mutations were inserted into $a m t R$ via two-step-PCR using the oligonucleotides amtR-PstI-rev, amtRw/oATGBamHI-fw and mutagenesis primers (Table 2). For amplification C. glutamicum ATCC13032 cell were used as a template. In the first step the mutagenesis primer was used as a forward primer, with amtR-PstI-rev as reverse primer. After denaturation at $96^{\circ} \mathrm{C}$ for $30 \mathrm{sec}$ the primers were aligned at $65^{\circ} \mathrm{C}$ for $30 \mathrm{sec}$ and elongation was performed at $72^{\circ} \mathrm{C}$ for $30 \mathrm{sec}$. The cycle was repeated 30 times. The resulting fragment served as a reverse primer in the second step, with amtRw/oATG-BamHI-fw as forward primer. Here $58^{\circ} \mathrm{C}$ was used as annealing temperature and elongation was carried out for $45 \mathrm{sec}$. The resulting fragment was cloned into the vector pMalc2 via the restriction sites BamHI and PstI. All plasmids constructed (Table 3) were sequenced for control.

\section{Protein purification}

E. coli BL21 [25] freshly transformed with pMalc2amtR* vectors was used for inoculation of $300 \mathrm{ml} \mathrm{LB}$ containing $2 \%$ glucose. Bacteria were grown over night at $37^{\circ} \mathrm{C}$ and used to inoculate $800 \mathrm{ml}$ fresh LB containing $2 \%$ glucose at an $\mathrm{OD}_{600}$ of 0.1. The culture was grown to an $\mathrm{OD}_{600}$ of 0.5 and subsequently induced with $0.3 \mathrm{mM}$ IPTG. After 4 $\mathrm{h}$ of incubation the cells were harvested $(3,000 \times \mathrm{g}, 10$ $\min , 4^{\circ} \mathrm{C}$ ) and the pellet was resuspended in $25 \mathrm{ml}$ purification buffer ( $20 \mathrm{mM}$ Tris-HCl, pH 7.4, $200 \mathrm{mM} \mathrm{NaCl}$, $1 \mathrm{mM}$ EDTA). The solution was sonicated three times for $30 \mathrm{sec}$ at 70\% (Bandelin Sonoplus UW2070, Berlin) and centrifugated for $10 \mathrm{~min}$ with $14,000 \times \mathrm{g}$ at $4{ }^{\circ} \mathrm{C}$. The supernatant was loaded onto a $1 \mathrm{ml}$ MBP-Trap column (GE Healthcare, Munich), washed with 10 column volumes purification buffer and protein bound was eluted with $20 \mathrm{mM}$ maltose in purification buffer.

\section{Gel retardation experiments}

Target DNA for gel shift assays was synthesized by PCR (for the primers used, see Table 2) and was purified by agarose gel electrophoresis. To label the DNA and to prepare the reaction mixture for the gel shift assay, the DIG gel shift kit (Roche, Mannheim) was used as recommended by the supplier. Separation by gel electrophoresis was performed in native $6 \%$ polyacrylamide gels (Anamed Electrophorese GmbH, Darmstadt, Germany) using $0.5 \times$ Tris-borate-EDTA buffer as the running buffer. Subsequently, the labelled DNA was transferred to a nylon membrane (Roche, Mannheim, Germany) by electro blotting as described in the protocol of the DIG gel shift kit (Roche, Mannheim, Germany). For detection of the labelled DNA, X-ray film was used.

\section{Preparation of amt $B_{p} D N A$ for $S P R$}

Thirty nucleotide synthetic oligonucleotides containing $a m t B_{\mathrm{p}}$ (forward: 5'TAAATTA CCTGTTAAACTATGAAAATATC; backward: 5'-GATATTTTCTATAGTTTAACAGG TAATTTA-3') or a nonspecific DNA sequence (5'CGCGATAATCTTTGCTAACCCTTT TGAGTT-3'; backward: 5'-AACTCAAAAGGGTTAGCAAAGATTATCGCG-3') were hybridized and used for analyses without further purification. The forward 30-nt oligonucleotides carried a biotin at the 3'-end. All oligonucleotides were purchased with or without modification from MWG Biotech (Ebersberg, Germany). The concentration of the hybridized DNA was determined using a pEQlab (Erlangen) Nanodrop Spectrophotometer.

\section{Surface plasmon resonance (SPR)}

SPR measurements with AmtR from C. glutamicum, overexpressed as maltose binding protein fusion in E. coli BL21, were performed using a BIAcoreX instrument operated at $25^{\circ} \mathrm{C}$ (Biacore, Uppsala, Sweden). For interaction analyses of an $a m t B$ promoter fragment with $A m t R$ and $A m t R$ variants 3 'end biotinylated DNA comprising $a m t B_{p}$ DNA sequence or a non-specific DNA sequence was immobilized on the surface of Biacore CM5 chips. For this purpose, the chip surface was activated with $35 \mu \mathrm{l}$ of a mixture of $50 \mathrm{mM}$ N-hydroxysuccinimide and $20 \mathrm{mM} \mathrm{N}$ - 
Table 3: Plasmids used in this study

\begin{tabular}{|c|c|c|}
\hline Plasmid & Genotype/Description & Reference \\
\hline pMalc2 & ptac, ApR, ori ColEI, malE, lacZ $\alpha$, lacla, E. coli-Vektor for protein purification & NEB, Schwalbach \\
\hline pMalc2amtR & PMalc2, $P_{\text {tac }}-$ malE-amtR & This work \\
\hline pMalc2amtR *Glu23Ala & pMalc2amtR, point mutation in $a m t R$ for AmtR*Glu23Ala variant & This work \\
\hline pMalc2amtR* Arg52Ala & pMalc2amtR, point mutation in $a m t R$ for AmtR*Arg52Ala variant & This work \\
\hline pMalc2amtR* Gly50Ala & pMalc2amtR, point mutation in $a m t R$ for AmtR*Gly50Ala variant & This work \\
\hline pMalc2amtR* Ile5IAla & pMalc2amtR, point mutation in amtR for AmtR*Ile5IAla variant & This work \\
\hline pMalc2amtR* Thr40Ala & pMalc2amtR, point mutation in amtR for AmtR*Thr40Ala variant & This work \\
\hline pMalc2amtR* Thr42Ala & pMalc2amtR, point mutation in $a m t R$ for AmtR*Thr42Ala variant & This work \\
\hline pMalc2amtR*Glu30Ala & pMalc2amtR, point mutation in $a m t R$ for AmtR*Glu30Ala variant & This work \\
\hline pMalc2amtR*Gly36Ala & pMalc2amtR, point mutation in $a m t R$ for AmtR*Gly36Ala variant & This work \\
\hline pMalc2amtR*Leu3 IAla & pMalc2amtR, point mutation in $a m t R$ for AmtR*Leu3।Ala variant & This work \\
\hline pMalc2amtR*Phe32Ala & pMalc2amtR, point mutation in amtR for AmtR*Phe32Ala variant & This work \\
\hline pMalc2amtR*Thr33Ala & pMalc2amtR, point mutation in $a m t R$ for AmtR*Thr33Ala variant & This work \\
\hline pMalc2amtRAla54Gly & pMalc2amtR, point mutation in $a m t R$ for AmtR*Ala54Gly variant & This work \\
\hline pMalc2amtRHis43Ala & pMalc2amtR, point mutation in $a m t R$ for AmtR*His43Ala variant & This work \\
\hline pMalc2amtR*Gly36Ala & pMalc2amtR, point mutation in $a m t R$ for AmtR*Gly36Ala variant & This work \\
\hline pMalc2amtR*Thr40Ala & pMalc2amtR, point mutation in $a m t R$ for AmtR*Thr40Ala variant & This work \\
\hline pMalc2amtR*Thr42Ala & pMalc2amtR, point mutation in amtR for AmtR*Thr42Ala variant & This work \\
\hline pMalc2amtR*Gly50Ala & pMalc2amtR, point mutation in $a m t R$ for AmtR*Gly50Ala variant & This work \\
\hline pMalc2amtR*lle5 IAla & pMalc2amtR, point mutation in $a m t R$ for AmtR*lle5 IAla variant & This work \\
\hline pMalc2amtR*Arg52Ala & pMalc2amtR, point mutation in $a m t R$ for AmtR*Arg52Ala variant & This work \\
\hline pMalc2amtR*Gln53Ala & pMalc2amtR, point mutation in $a m t R$ for AmtR*Glu53Ala variant & This work \\
\hline pMalc2amtR*Ser55Ala & pMalc2amtR, point mutation in $a m t R$ for AmtR*Ser55Ala variant & This work \\
\hline pMalc2amtR*Leu56Ala & pMalc2amtR, point mutation in $a m t R$ for AmtR*Leu56Ala variant & This work \\
\hline pMalc2amtR*Tyr57Ala & pMalc2amtR, point mutation in $a m t R$ for AmtR*Tyr57Ala variant & This work \\
\hline pMalc2amtR*Tyr58Ala & pMalc2amtR, point mutation in $a m t R$ for AmtR*Tyr58Ala variant & This work \\
\hline pMalc2amtR*His59Ala & pMalc2amtR, point mutation in $a m t R$ for AmtR*His59Ala variant & This work \\
\hline pMalc2amtR*Leu70Ala & pMalc2amtR, point mutation in amtR for AmtR*Leu70Ala variant & This work \\
\hline pMalc2amtR*Leu7IAla & pMalc2amtR, point mutation in $a m t R$ for AmtR*Leu7IAla variant & This work \\
\hline
\end{tabular}


ethyl-N-(3-dimethylaminopropyl)-carbodiimide-hydrochloride (Biacore, Uppsala, Schweden). After coupling of 3,000 RU neutravidine ( $5 \mu \mathrm{M}$ in $10 \mathrm{mM} \mathrm{Na-acetate,} \mathrm{pH}$ 5.0), to the chip, $35 \mu \mathrm{l} 1 \mathrm{M}$ ethanolamine (Biacore, Uppsala, Sweden) was used to inactivate the remaining reactive carboxyl groups on the chip. Hybridized non-specific DNA was coupled in flow cell 1 and $a m t B_{p}$ DNA in flow cell 2. In all measurements HBS-EP was used as a running buffer. The flow rate was $5 \mu \mathrm{l} / \mathrm{min}$ during coupling and 40 $\mu \mathrm{l} / \mathrm{min}$ for all measurements. To regenerate the chip surface the dissociation of the $a m t B_{\mathrm{P}}-\mathrm{AmtR}$ or $\mathrm{amtB}_{\mathrm{P}}-\mathrm{AmtR}^{*}$ variants complex was stopped by injection of $80 \mu \mathrm{l}$ HBSEP buffer at $40 \mu \mathrm{l} / \mathrm{min}$ after each injection. AmtR ${ }^{*}$-MBP variant concentrations of $2170 \mathrm{nM}, 10870 \mathrm{nM}, 28260 \mathrm{nM}$ and $43470 \mathrm{nM}$ were used. Additionally $43 \mathrm{nM}$ and 870 $\mathrm{nM}$ of AmtR were used for the SPR measurements. Evaluation of the data was performed using BiaEvaluation 4.0 Software (Biacore, Uppsala, Schweden). The titrations for the kinetic measurements have been carried out twice for each AmtR variant.

\section{Model building}

The structure of AmtR from C. glutamicum in complex with DNA was modeled based on the crystal structure of the TetR repressor/operator complex from E. coli (PDB code: 1QPI) [18]. Both proteins share a sequence identity of $37 \%$ in the DNA binding domain, which is also reflected in the significant $\mathrm{E}$-value of $10^{-5}$ for the respective sequence alignment. Since the remaining parts of the two repressors are highly divergent in sequence, modeling was restricted to the DNA binding domain. The two DNA binding heads of dimeric AmtR were modeled separately using Swiss-Model [26] and the structure of the protein DNA complex was obtained by assuming an identical interface geometry as in the TetR-DNA complex.

The model was subsequently refined by 100 steps of energy minimization using the Sybyl 7.3 program package (Tripos Inc.). The quality of the structure was assessed using Procheck [27] and Whatcheck [28] and did not reveal any steric clashes or unfavorable geometries thus confirming the overall good quality of the model. Finally, contacts between the AmtR and its target DNA were retrieved using LIGPLOT [29]. Visualization and analysis of the model features were carried out using the program Discovery Studio Visualizer (Accelrys Software Inc.).

\section{Authors' contributions}

$\mathrm{DM}, \mathrm{NJ}$ and $\mathrm{KH}$ carried out cloning, site-directed mutagenesis experiments, protein purifications, gel retardation assays and SPR. Molecular modelling was carried out CJ. $\mathrm{HS}$ and $\mathrm{AB}$ conceived the study and participated in its design and coordination and drafted the manuscript. All authors read and approved the final manuscript.

\section{Acknowledgements}

This work was supported by the Deutsche Forschungsgemeinschaft (SFB 473, projects $\mathrm{ClO}$ and $\mathrm{Cl}$ 2). NJ was supported by a fellowship of the Universität Bayern. The authors thank J. Amon (Erlangen) for the preparation of Fig. I.

\section{References}

I. Takors R, Bathe B, Rieping M, Hans S, Kelle R, Huthmacher K: Systems biology for industrial strains and fermentation processes - Example: Amino acids. J Biotechnol 2007, 129: I8I-190.

2. Burkovski A: Nitrogen metabolism and its regulation. In Handbook of Corynebacterium glutamicum Edited by: Bott M, Eggeling L. Boca Raton: CRC Press LLC; 2005:333-349.

3. Burkovski A: Nitrogen control in Corynebacterium glutamicum: proteins, mechanisms, signals. J Microbiol Biotechnol 2007, 17:187-194.

4. Hänßler E, Burkovski A: Molecular mechanisms of nitrogen control in corynebacteria. In Corynebacteria: genomics and molecular biology Edited by: Burkovski A. Norfolk: Caister Academic Press; 2008: 183-20I.

5. Jakoby M, Nolden L, Meier-Wagner J, Krämer R, Burkovski A: AmtR, a global repressor in the nitrogen regulation system of Corynebacterium glutamicum. Mol Microbiol 2000, 37:964-977.

6. Walter B, Hänßler E, Kalinowski J, Burkovski A: Nitrogen metabolism and nitrogen control in corynebacteria: variations of a common theme. J Mol Microbiol Biotechnol 2007, I 2: I3 I-I 38.

7. Beckers G, Strösser J, Hildebrandt U, Kalinowski J, Farwick M, Krämer R, Burkovski A: Regulation of AmtR-controlled gene expression in Corynebacterium glutamicum: mechanism and characterization of the AmtR regulon. Mol Microbiol 2005, 58:580-595.

8. Buchinger S, Strösser J, Rehm N, Hänßler E, Hans S, Bathe B, Schomburg D, Krämer R, Burkovski A: A combination of transcriptome and metabolome analyses reveals new targets of the Corynebacterium glutamicum nitrogen regulator AmtR. J Biotechnol 2009, 140:68-74.

9. Ramos JL, Martinez-Bueno M, Molina-Henares AJ, Teran W, Watanabe K, Zhang X, Gallegos MT, Brennan R, Tobes R: The TetR family of transcriptional repressors. Microbiol Mol Biol Rev 2005, 69:326-356.

10. Resch M, Striegl H, Henssler EM, Sevvana M, Egerer-Sieber C, Schiltz $E$, Hillen W, Muller YA: A protein functional leap: how a single mutation reverses the function of the transcription regulator TetR. Nucleic Acids Res 2008, 36:4390-440I.

II. Silberbach M, Hüser A, Kalinowski J, Pühler A, Walter B, Krämer R, Burkovski $A$ : DNA microarray analysis of the nitrogen starvation response of Corynebacterium glutamicum. J Biotechnol 2005, I I 9:357-367.

12. Silberbach M, Schäfer M, Hüser A, Kalinowski J, Pühler A, Krämer R, Burkovski A: Adaptation of Corynebacterium glutamicum to ammonium-limitation: a global analysis using transcriptome and proteome techniques. Appl Environ Microbiol 2005, 71:2391-2402.

13. Brune I, Brinkrolf K, Kalinowski J, Pühler A, Tauch A: The individual and common repertoire of DNA-binding transcriptional regulators of Corynebacterium glutamicum, Corynebacterium efficiens, Corynebacterium diphtheriae and Corynebacterium jeikeium deduced from the complete genome sequences. BMC Genomics 2005, 6:86.

14. Krug A, Wendisch VF, Bott M: Identification of AcnR, a TetRtype repressor of the aconitase gene acn in Corynebacterium glutamicum. J Biol Chem 2005, 280:585-595.

15. Itou $\mathrm{H}$, Okada U, Suzuki $\mathrm{H}$, Yao M, Wachi M, Watanabe $\mathrm{N}$, Tanaka I: The CGL26 I 2 protein from Corynebacterium glutamicum is a drug resistance-related transcriptional repressor. J Biol Chem 2005, 280:387II-387/9.

16. Rey D, Pühler A, Kalinowski J: The putative transcriptional repressor McbR, member of the TetR-family, is involved in the regulation of the metabolic network directing the synthesis of sulphur containing amino acids in Corynebacterium glutamicum. J Biotechnol 2003, 103:5 I-65.

17. Rey D, Nentwich SS, Koch DJ, Rückert C, Pühler A, Tauch A, Kalinowski J: The McbR repressor modulated by the effector substance $S$-adenosylhomocysteine controls directly the transcription of a regulon involved in sulphur metabolism of 
Corynebacterium glutamicum ATCC 13032. Mol Microbiol 2005, 56:87I-887.

18. Orth P, Schnappinger D, Hillen W, Saenger W, Hinrichs W: Structural basis of gene regulation by the tetracyclin inducible Tet repressor-operator system. Nat Struct Biol 2000, 7:215-219.

19. Helbl V, Hillen W: Stepwise selection of TetR variants recognizing tet operator $4 \mathrm{C}$ with high affinity and specificity. J Mol Biol 1998, 276:313-318.

20. Forchhammer $K: \mathbf{P}_{\|}$signal transducers: novel functional and structural insights. Trends Microbiol 2008, 16:65-72.

21. Abe S, Takayama K, Kinoshita S: Taxonomical studies on glutamic acid-producing bacteria. J Gen Microbiol 1967, |3:279-30|.

22. Keilhauer C, Eggeling L, Sahm H: Isoleucine synthesis in Corynebacterium glutamicum: molecular analysis of the ilvB-ilvN-ilvC operon. J Bacteriol 1993, 175:5595-5603.

23. Sambrook J, Fritsch EF, Maniatis T: Molecular Cloning: a Laboratory Manual 2nd edition. Cold Spring Habor NY: Cold Spring Habor Laboratory; 1989.

24. Grant SNG, Jessee J, Bloom FR, Hanahan D: Differential plasmid rescue from transgenic mouse DNAs into Escherichia coli methylation-restriction mutants. Proc Natl Acad Sci USA 1990, 87:4645-4649.

25. Studier FW, Rosenberg AH, Dunn JJ, Dubendorff JW: Use of T7 polymerase to direct expression of cloned genes. In Methods Enzymol Volume 185. Academic Press, San Diego, CA; 1990:60-89.

26. Guex N, Peitsch MC: SWISS-MODEL and the Swiss-PdbViewer: an environment for comparative protein modeling. Electrophoresis 1997, I 8:27|4-2723.

27. Laskowski RA, Moss DS, Thornton JM: Main-chain bond lengths and bond angles in protein structures. I Mol Biol 1993, 23 I: $1049-1067$.

28. Hooft RW, Vriend G, Sander C, Abola EE: Errors in protein structures. Nature 1996, 381:272.

29. Wallace AC, Laskowski RA, Thornton JM: LIGPLOT: a program to generate schematixc diagrams of protein-ligand interactions. Protein Engineering 1995, 1995:127-134.

Publish with Biomed Central and every scientist can read your work free of charge

"BioMed Central will be the most significant development for disseminating the results of biomedical research in our lifetime. "

Sir Paul Nurse, Cancer Research UK

Your research papers will be:

- available free of charge to the entire biomedical community

- peer reviewed and published immediately upon acceptance

- cited in PubMed and archived on PubMed Central

- yours - you keep the copyright 